\title{
Mixing actions of groups with the Haagerup approximation property
}

by

\author{
Greg Hjorth (Melbourne)
}

\begin{abstract}
A countable group $\Gamma$ has the Haagerup approximation property if and only if the mixing actions are dense in the space of all actions of $\Gamma$.
\end{abstract}

1. Introduction. Given a countable group $\Gamma$ and an atomless standard Borel probability space $(X, \mu)$, we can form the space of all measure preserving actions of $\Gamma$. In the usual weak topology, as discussed below, this becomes a Polish space. In the lines below, every action is assumed to be measurable, measure preserving, on some such $(X, \mu)$.

The present paper is part of a series of results which relate representation-theoretic properties of a group to the properties of its space of all actions on some such $(X, \mu)$.

THEOREM 1.1 (Ornstein-Weiss; [9]). A countable group $\Gamma$ is amenable if and only if every free measure preserving action has almost invariant sets.

Here, we say that an action of $\Gamma$ on $(X, \mu)$ has almost invariant sets if there exists a sequence of measurable sets, $\left(A_{n}\right)_{n \in \mathbb{N}}$, with measure bounded away from 0 and 1 , such that for any $\gamma \in \Gamma$,

$$
\limsup _{n \rightarrow \infty} \mu\left(A_{n} \triangle \gamma \cdot A_{n}\right)=0
$$

Theorem 1.2 (Connes-Weiss, Schmidt; [3], [11]). A countable group $\Gamma$ has property $(\mathrm{T})$ if and only if no ergodic action has almost invariant sets.

TheOREM 1.3 (Glasner-Weiss; [4]). A countable group $\Gamma$ has property (T) if and only if the set of ergodic actions is closed in the space of all actions.

2000 Mathematics Subject Classification: Primary 03E15, 37A25.

Key words and phrases: Haagerup approximation property, mixing action, ergodic equivlaence relations. 
In answer to a question of Bergelson and Rosenblatt:

Theorem 1.4 (Kerr-Pichot; [8]). A countable group $\Gamma$ does not have property $(\mathrm{T})$ if and only if the weak mixing actions on $(X, \mu)$ are a dense $G_{\delta}$ in the space of all actions.

In the present paper we answer a question due to Alexander Kechris [7] by showing:

THEOREM 1.5. If a countable group $\Gamma$ has the Haagerup approximation property $(H A P)$ then the mixing actions are dense in the space of all actions.

The converse direction was known and is a straightforward consequence of the definitions - see for instance [7, 12.7]. Thus we have

COROLlary 1.6. A countable group $\Gamma$ has the Haagerup approximation property if and only if the mixing actions are dense in the space of all actions.

2. Representation-theoretic notions. For the reader's convenience we recall the relevant notions arising from the theory of unitary representations.

Definition. For $H$ a Hilbert space, we let $U(H)$ be the unitary operators. Given a unitary representation

$$
\pi: \Gamma \rightarrow U(H), \quad \gamma \mapsto \pi_{\gamma},
$$

of a countable group $\Gamma$, we say that $\pi$ has almost invariant vectors if for all $\varepsilon>0$ and $F \subset \Gamma$ finite there exists $v \in H$ with $\|v\|=1$, and for all $\gamma \in F$,

$$
\left\|\pi_{\gamma}(v)-v\right\|<\varepsilon .
$$

We say that the representation is mixing if for all $u, v \in H$,

$$
\limsup _{\gamma \rightarrow \infty}\left\langle\pi_{\gamma}(v), u\right\rangle=0 .
$$

In other words, for all $\varepsilon>0$ there exists a finite set $F \subset \Gamma$ such that for all $\gamma \in \Gamma \backslash F$,

$$
\left|\left\langle\pi_{\gamma}(v), u\right\rangle\right|<\varepsilon .
$$

Definition. Given a countable group $\Gamma$, we define the left regular representation of $\Gamma$ by

$$
\lambda: \Gamma \rightarrow \ell^{2}(\Gamma), \quad \gamma \mapsto \lambda_{\gamma},
$$

where

$$
\left(\lambda_{\gamma}(f)\right)(x)=f\left(\gamma^{-1} x\right) .
$$

With these concepts, we can define the classes of groups from the introduction. Note that the definition we give for amenability is equivalent to the more traditional definitions; there are manifold different characterizations of amenability, most of which, including the one used here, are discussed in $[10]$. 
Definition. A countable group $\Gamma$ is amenable if the left regular representation has almost invariant vectors. A countable group $\Gamma$ has property $(\mathrm{T})$ if whenever a unitary representation has almost invariant vectors, there is an invariant vector of norm one. A countable group has the Haagerup approximation property, HAP for short, if there exists a mixing action with almost invariant vectors.

ExAmples. (i) The group $\mathbb{Z}$ is amenable, as is any abelian group. More generally, any solvable group is amenable. See [10].

(ii) $\mathbb{F}_{2}$, the free group on two generators, is non-amenable, but it does have the Haagerup approximation property. See the appendix of [6] for an entirely elementary proof of this fact first due to Haagerup.

(iii) $\mathrm{SL}_{3}(\mathbb{Z})$ has property $(\mathrm{T})$. See for instance [5]. It is not amenable, since no countably infinite group can have an invariant unit vector in its left regular representation. Similarly, it does not have HAP, since mixing actions cannot have invariant unit vectors.

(iv) Under the natural semidirect product provided by linear action of $\mathrm{SL}_{2}(\mathbb{Z})$ on $\mathbb{Z}^{2}$, the group

$$
\Delta=\mathrm{SL}_{2}(\mathbb{Z}) \ltimes \mathbb{Z}^{2}
$$

is non-amenable, since it has a subgroup isomorphic to $\mathbb{F}_{2}$. It does not have property $(\mathrm{T})$, since it has $\mathrm{SL}_{2}(\mathbb{Z})$ as a homomorphic image, and this group in turn has the free group on two generators as a finite index subgroup. On the other hand, as shown for example in [2], it does have a kind of relative property $(\mathrm{T})$, which is sufficient to exclude the Haagerup approximation property.

3. Measure-theoretic notions. We follow the notation of [7].

Notation. For $(X, \mu)$ a standard Borel space, we let $\operatorname{Aut}(X, \mu)$ be the collection of invertible measure preserving transformations. Given a countable group $\Gamma, A(\Gamma, X, \mu)$ denotes the collection of homomorphisms from $\Gamma$ to $\operatorname{Aut}(X, \mu)$. Given such an automorphism

$$
a: \Gamma \rightarrow \operatorname{Aut}(X, \mu)
$$

and a group element $\gamma \in \Gamma$, we denote by $\gamma^{a}$ the invertible measure preserving transformation associated to $\gamma$. In the rare cases below that there can be no possible confusion regarding which action we have in mind, we will simply write $\gamma$ instead of $\gamma^{a}$, and $\gamma \cdot x$ instead of $\gamma^{a} \cdot x$.

Notation. The weak topology on $\operatorname{Aut}(X, \mu)$ is the topology with subbasic open sets of the form

$$
\{\pi \in \operatorname{Aut}(X, \mu): \mu(B \triangle \pi(A))<\varepsilon\}
$$

for $A, B \subset X$ measurable, $\varepsilon>0$. 
Provided we identify measure preserving transformations which agree on a conull set, $\operatorname{Aut}(X, \mu)$ becomes a Polish space in the weak topology. Then $A(\Gamma, X, \mu)$ becomes a closed subset of

$$
\prod_{\Gamma} \operatorname{Aut}(X, \mu)
$$

and is again Polish in its own right. These and other preliminary facts can be found at $\S 1$ and $\S 10$ of [7].

Definition. Given a countable group $\Gamma$ and an action $a \in A(\Gamma, X, \mu)$, we say that the action is mixing if for all measurable $A, B \subset X$,

$$
\limsup _{\gamma \rightarrow \infty}\left|\mu\left(A \triangle \gamma^{a} \cdot B\right)-\mu(A) \mu(B)\right|=0 .
$$

We will need a non-trivial, but well known, consequence of the Haagerup approximation property.

Definition. Let $E$ and $F$ be equivalence relations on standard Borel spaces $X$ and $Y$. A homomorphism from $E$ to $F$ is a function $\theta: X \rightarrow Y$ such that for all $x_{1}, x_{2} \in X$ with $x_{1} E x_{2}$ we have

$$
\theta\left(x_{1}\right) F \theta\left(x_{2}\right) .
$$

Definition. $E_{0}$ is the equivalence relation of eventual agreement on infinite binary sequences: thus, for $f, g \in\{0,1\}^{\mathbb{N}}=\prod_{\mathbb{N}}\{0,1\}$, we set $f E_{0} g$ if there exists some $N \in \mathbb{N}$ such that

$$
(\forall n>N)(f(n)=g(n)) .
$$

Theorem 3.1 (Jolissaint, see for instance $[7,11.1]$ ). A countable group $\Gamma$ has the Haagerup approximation property if and only if it has a free, measure preserving, mixing action on a standard Borel probability space which is not $E_{0}$-ergodic.

COROLlaRY 3.2. If $\Gamma$ has the Haagerup approximation property, then for any finite $F \subset \Gamma$ we can find a standard Borel probability space, $(Y, \nu)$, which, for any $\delta>0$, has a partition

$$
Y=\bigcup_{i \leq N} A_{i}
$$

and an action of $\Gamma$ on $Y$ such that:

(i) the action is measure preserving and mixing;

(ii) for each $\gamma \in F$ and $i \leq N$,

$$
\nu\left(A_{i} \triangle \gamma \cdot A_{i}\right)<\delta
$$

(iii) each $A_{i}$ has measure $1 / N$. 
Proof. Take as our $(Y, \nu)$ the witness that $\Gamma$ has a mixing, measure preserving action which is not $E_{0}$-ergodic. Let $\theta: Y \rightarrow 2^{\mathbb{N}}$ be the function witnessing failure of $E_{0}$-ergodicity.

First we choose some large $M$ such that for all $\gamma \in F$,

$$
\nu(\{y \in Y:(\exists n \geq M)(\theta(y)(n) \neq \theta(\gamma \cdot y)(n))\})<\delta .
$$

Then we define

$$
\varrho: Y \rightarrow 2^{\mathbb{N}} \text { by } \varrho(y)(n)=\theta(y)(M+n) .
$$

Rephrasing our assumption on $M$ we see that for each $\gamma \in F$,

$$
\nu(\{y \in Y: \varrho(y) \neq \varrho(\gamma \cdot y)\})<\delta .
$$

The assumptions on $\theta$ imply that the pullback of every point in $2^{\mathbb{N}}$ under $\theta$ is $\nu$-null, and hence the pullback of every point under $\varrho$ is $\nu$-null.

Define $\mu=\varrho^{*}(\nu)$ on $2^{\mathbb{N}}$ by $\mu(A)=\nu\left(\varrho^{-1}[A]\right)$. The previous remark implies that this measure is continuous - that is, no point has non-zero measure. Now if we partition $2^{\mathbb{N}}$ into sets $B_{1}, \ldots, B_{N}$, each of $\mu$-measure $1 / N$, then we can finish with $A_{i}=\theta^{-1}\left[B_{i}\right]$.

There is one further piece of notation which we will find convenient to use.

Notation. Given $A \subset X \times Y$, we let

$$
\begin{array}{ll}
A_{x}=\{y \in Y:(x, y) \in A\} & \text { for } x \in X, \\
A^{y}=\{x \in X:(x, y) \in A\} & \text { for } y \in Y .
\end{array}
$$

4. Proof. In everything below, $\Gamma$ is a countable group with the Haagerup approximation property. The next lemma, with its cutting and splicing along almost invariant sets, has obvious parallels with the arguments of [8].

Lemma 4.1. Let $(X, \mu)$ be an atomless standard Borel probability space. Let $a \in A(\Gamma, X, \mu), B_{1}, \ldots, B_{n}$ be measurable subsets of $X, F \subset \Gamma$ finite, and $\varepsilon>0$. Then there exists an atomless standard Borel probability space $(\widehat{X}, \widehat{\mu})$ and $b \in A(\Gamma, \widehat{X}, \widehat{\mu})$ and measurable subsets $\widehat{B}_{1}, \ldots, \widehat{B}_{n}$ such that:

(a) for any $S \subset\{1, \ldots, n\}$,

$$
\widehat{\mu}\left(\bigcap_{i \in S}\left(\widehat{B}_{i} \backslash \bigcap_{i \notin S} \widehat{B}_{i}\right)\right)=\mu\left(\bigcap_{i \in S}\left(B_{i} \backslash \bigcap_{i \notin S} B_{i}\right)\right) ;
$$

(b) for all $m \leq n$ and $\gamma_{1}, \ldots, \gamma_{m} \in F$ and $i_{1}, \ldots, i_{m} \leq n$,

$$
\left|\mu\left(\gamma_{1}^{a} \cdot B_{i_{1}} \cap \cdots \cap \gamma_{m}^{a} \cdot B_{i_{m}}\right)-\widehat{\mu}\left(\gamma_{1}^{b} \cdot \widehat{B}_{i_{1}} \cap \cdots \cap \gamma_{m}^{b} \cdot \widehat{B}_{i_{m}}\right)\right|<\varepsilon ;
$$

(c) for all $\gamma \in \Gamma$ and $i, j \leq n$,

$$
\left|\widehat{\mu}\left(\widehat{B}_{i} \cap \gamma^{b} \cdot \widehat{B}_{j}\right)-\widehat{\mu}\left(\widehat{B}_{i}\right) \cdot \widehat{\mu}\left(\widehat{B}_{j}\right)\right| \leq\left|\mu\left(B_{i} \cap \gamma^{a} \cdot B_{j}\right)-\mu\left(B_{i}\right) \cdot \mu\left(B_{j}\right)\right| ;
$$


(d) for all $i, j \leq n$,

$$
\limsup _{\gamma \rightarrow \infty}\left|\widehat{\mu}\left(\gamma^{b} \cdot \widehat{B}_{i} \cap \widehat{B}_{j}\right)-\widehat{\mu}\left(\widehat{B}_{i}\right) \widehat{\mu}\left(\widehat{B}_{j}\right)\right|<\varepsilon .
$$

Proof. To begin, let us fix some large $N \in \mathbb{N}$. (It will be clear after the later calculations that any sufficiently large $N$ will work; in fact, $N>2 / \varepsilon$ suffices.) We equip $X^{N}$ with the diagonal action, $c$, where

$$
\gamma^{c} \cdot\left(x_{1}, \ldots, x_{N}\right)=\left(\gamma^{a} \cdot x_{1}, \ldots, \gamma^{a} \cdot x_{N}\right)
$$

and the product measure $\mu^{N}$. For each $i \leq n$ we let

$$
\pi_{i}: X^{N} \rightarrow X, \quad\left(x_{1}, \ldots, x_{N}\right) \mapsto x_{i} .
$$

Now let $\delta>0$ be very small. (Again, it will be clear from the later calculations that any sufficiently large $\delta$ will fill our needs; in fact, $\delta<\varepsilon / N$ suffices.) Keeping in mind that $\Gamma$ has HAP as at 3.2 , let $(Y, \nu)$ be a standard Borel probability space with a partition

$$
Y=\bigcup_{i \leq N} A_{i}
$$

and an action $d$ of $\Gamma$ on $Y$ such that:

(i) the action $d$ is measure preserving and mixing;

(ii) for each $\gamma \in F$ and $i \leq n$,

$$
\nu\left(A_{i} \triangle \gamma^{d} \cdot A_{i}\right)<\delta
$$

(iii) each $\nu\left(A_{i}\right)$ equals $1 / N$.

We let $\widehat{X}=X^{N} \times Y$, equipped with the product measure, which we now denote by $\widehat{\mu}$, and the action

$$
\gamma^{b} \cdot(\vec{x}, y)=\left(\gamma^{c} \cdot \vec{x}, \gamma^{d} \cdot y\right) .
$$

We then let $\widehat{B}_{j}$ be the set of $(\vec{x}, y) \in X^{N} \times Y$ such that for all $i \leq N$,

$$
y \in A_{i} \Rightarrow \pi_{i}(\vec{x}) \in B_{j} .
$$

Property (a) from the lemma should be obvious from the construction. Property (b) follows by making $\delta$ sufficiently small.

For each $\gamma \in \Gamma$ we let

$$
C_{\gamma}=\left\{y \in Y:(\forall i)\left(y \in A_{i} \Rightarrow \gamma^{d} \cdot y \notin A_{i}\right)\right\} .
$$

Since the action of $d$ is mixing we deduce that, as $\gamma \rightarrow \infty$,

and hence

$$
\nu\left(A_{i} \cap\left(\gamma^{d}\right)^{-1} \cdot\left(Y \backslash A_{i}\right)\right) \rightarrow \frac{N-1}{N^{2}},
$$

$$
\nu\left(C_{\gamma}\right) \rightarrow \frac{N(N-1)}{N^{2}}=\frac{N-1}{N} .
$$


Claim. For all $\gamma$ outside a finite set, and for all $j, l \leq n$,

$$
\frac{N-2}{N} \mu\left(B_{j}\right) \cdot \mu\left(B_{l}\right)<\widehat{\mu}\left(\widehat{B}_{j} \cap \gamma^{b} \cdot \widehat{B}_{l}\right)<\frac{N-2}{N} \mu\left(B_{j}\right) \cdot \mu\left(B_{l}\right)+\frac{2}{N} .
$$

Proof of Claim. Outside a finite set of $\gamma$ 's we obtain $\nu\left(C_{\gamma}\right)>(N-2) / N$. Then for $y \in C_{\gamma}, y \in A_{i}, \gamma^{d} \cdot y \in A_{k}, z \in A_{k}$ we find firstly that $i \neq k$ and then

$$
\begin{aligned}
\mu^{N}\left(\left(\widehat{B}_{j}\right)^{z} \cap\left(\gamma^{b} \cdot \widehat{B}_{l}\right)^{\gamma^{d} \cdot y}\right) & =\mu^{N}\left(\left(\widehat{B}_{j}\right)^{z} \cap\left(\gamma^{c} \cdot\left(\widehat{B}_{l}\right)^{y}\right)\right) \\
& =\mu\left(\pi_{k}^{-1}\left(B_{j}\right) \cap \pi_{i}^{-1}\left(\gamma^{a} \cdot B_{l}\right)\right)=\mu\left(B_{j}\right) \cdot \mu\left(B_{l}\right) .
\end{aligned}
$$

And thus for $z \in \gamma^{d} \cdot C_{\gamma}$ we have

$$
\mu^{N}\left(\left(\widehat{B}_{j}\right)^{z} \cap\left(\gamma^{b} \cdot \widehat{B}_{l}\right)^{z}\right)=\mu\left(B_{j}\right) \cdot \mu\left(B_{l}\right) .
$$

For $z$ outside this set,

$$
0 \leq \mu^{N}\left(\left(\widehat{B}_{j}\right)^{z} \cap\left(\gamma^{b} \cdot \widehat{B}_{l}\right)^{z}\right) \leq 1 .
$$

Since we have $\nu\left(C_{\gamma}\right)=\nu\left(\gamma^{d} \cdot C_{\gamma}\right)>(N-2) / N$ the claim follows by integrating $z$ over $Y$. Claim

Thus for $N$ with $2 / N<\varepsilon$ we obtain (d) from the statement of the lemma.

Note then that for all $i, l \leq n$ and $\gamma \in \Gamma$ we have, as above,

$$
\widehat{\mu}\left(\widehat{B}_{i} \cap \gamma^{b} \cdot\left(\widehat{B}_{l}\right)\right)=\int_{Y} \mu^{N}\left(\left(\widehat{B}_{i}\right)^{z} \cap\left(\gamma^{b} \cdot \widehat{B}_{l}\right)^{z} d \nu(z) .\right.
$$

For $z \in \gamma^{d} \cdot C_{\gamma}$ again

$$
\mu^{N}\left(\left(\widehat{B}_{j}\right)^{z} \cap\left(\gamma^{b} \cdot \widehat{B}_{l}\right)^{z}\right)=\mu\left(B_{j}\right) \cdot \mu\left(B_{l}\right),
$$

whilst for $z \notin \gamma^{d} \cdot C_{\gamma}$ we obtain

$$
\mu^{N}\left(\left(\widehat{B}_{j}\right)^{z} \cap\left(\gamma^{b} \cdot \widehat{B}_{l}\right)^{z}\right)=\mu\left(B_{j} \cap \gamma^{a} \cdot B_{l}\right) .
$$

This yields (c).

Corollary 4.2. Let $(X, \mu)$ be an atomless standard Borel probability space. Let $a \in A(\Gamma, X, \mu), C_{1}, \ldots, C_{k}$ be measurable subsets of $X, F \subset \Gamma$ finite, and $\varepsilon>0$. Then there exists $c \in A(\Gamma, X, \mu)$ such that:

(i) for all $m \leq k$ and $\gamma_{1}, \ldots, \gamma_{m} \in F$ and $i_{1}, \ldots, i_{m} \leq k$,

$$
\left|\mu\left(\left(\gamma_{1}^{a} \cdot C_{i_{1}} \cap \cdots \cap \gamma_{m}^{a} \cdot C_{i_{m}}\right) \triangle\left(\gamma_{1}^{c} \cdot C_{i_{1}} \cap \cdots \cap \gamma_{m}^{c} \cdot C_{i_{m}}\right)\right)\right|<\varepsilon ;
$$

(ii) for all $\gamma \in \Gamma$ and $i, j \leq n$

$$
\left|\mu\left(C_{i} \cap \gamma^{c} \cdot C_{j}\right)-\mu\left(C_{i}\right) \cdot \mu\left(C_{j}\right)\right| \leq\left|\mu\left(C_{i} \cap \gamma^{a} \cdot C_{j}\right)-\mu\left(C_{i}\right) \cdot \mu\left(C_{j}\right)\right| ;
$$

(iii) for all $i, j \leq n$,

$$
\limsup _{\gamma \rightarrow \infty}\left|\mu\left(\gamma^{c} \cdot C_{i} \cap C_{j}\right)-\mu\left(C_{i}\right) \cdot \mu\left(C_{j}\right)\right|<\varepsilon .
$$


Proof. Let $\mathcal{B}$ be the finite Boolean algebra generated by $\left\{\gamma \cdot C_{i}: i \leq k\right.$, $\gamma \in F\}$. Let $B_{1}, \ldots, B_{n}$ enumerate the elements of $\mathcal{B}$. Apply the last lemma to obtain $b$ and $(\widehat{X}, \widehat{\mu})$ for $B_{1}, \ldots, B_{n}, a \in A(\Gamma, X, \mu), \varepsilon>0$, and the indicated $F$.

Now we can find a measure preserving bijection $\psi: \widehat{X} \rightarrow X$ with $\psi\left[\widehat{B}_{i}\right]=B_{i}$. Taking

$$
\gamma^{c} \cdot x=\psi\left(\gamma^{b} \cdot \psi^{-1}(x)\right)
$$

is as required.

TheOREM 4.3. Let $\Gamma$ be a countable group with the Haagerup approximation property. Let $(X, \mu)$ be an atomless standard Borel probability space. Then the mixing actions are dense in the space $A(\Gamma, X, \mu)$.

Proof. Fix $a \in A(\Gamma, X, \mu)$. Fix $\varepsilon>0$. Fix $\mathcal{B}$ a finite Boolean algebra of measurable subsets of $X$. Fix a finite subset $F$ of $\Gamma$. We need to find a mixing action $b$ of $\Gamma$ such that for all $A \in \mathcal{B}$ and all $\gamma \in F$,

$$
\mu\left(\gamma^{b} \cdot A \triangle \gamma^{a} \cdot A\right)<\varepsilon .
$$

Let $\mathcal{B}_{0}=\mathcal{B} \subset \mathcal{B}_{1} \subset \mathcal{B}_{2} \subset \cdots$ be an increasing sequence of finite Boolean algebras of measurable subsets of $X$ whose union is dense in $M(X, \mu)$, the metric space of all measurable subsets of $X$ equipped with the metric $d(A, B)=\mu(A \triangle B)$. Let $F_{0}=F \subset F_{1} \subset F_{2} \subset \cdots$ be an increasing sequence of finite subsets of $\Gamma$ whose union equals all of $\Gamma$.

Applying the above corollary repeatedly we can find actions

$$
a_{0}=a, a_{1}, a_{2}, \ldots
$$

such that for each $n \geq 0$ and $C_{1}, \ldots, C_{k} \in \mathcal{B}_{n}$ we have:

(i) for all $m \leq k$ and $\gamma_{1}, \ldots, \gamma_{k} \in F_{n}$,

$$
\left|\mu\left(\left(\gamma_{1}^{a_{n}} \cdot C_{1} \cap \cdots \cap \gamma_{k}^{a_{n}} \cdot C_{k}\right) \triangle\left(\gamma_{1}^{a_{n+1}} \cdot C_{1} \cap \cdots \cap \gamma_{k}^{a_{n+1}} \cdot C_{k}\right)\right)\right|<2^{-n-1} \varepsilon ;
$$

(ii) for all $\gamma \in \Gamma$ and $i, j \leq k$,

$$
\left|\mu\left(C_{i} \cap \gamma^{a_{n+1}} \cdot C_{j}\right)-\mu\left(C_{i}\right) \cdot \mu\left(C_{j}\right)\right| \leq\left|\mu\left(C_{i} \cap \gamma^{a_{n}} \cdot C_{j}\right)-\mu\left(C_{i}\right) \cdot \mu\left(C_{j}\right)\right| ;
$$

(iii) for all $i, j \leq k$,

$$
\limsup _{\gamma \rightarrow \infty}\left|\mu\left(\gamma^{a_{n+1}} \cdot C_{i} \cap C_{j}\right)-\mu\left(C_{i}\right) \cdot \mu\left(C_{j}\right)\right|<2^{-n-1} \varepsilon .
$$

For each $\gamma \in \Gamma$ we can define the corresponding sequence of transformations

$$
\psi_{n}^{\gamma}: x \mapsto \gamma^{a_{n}} \cdot x .
$$

In light of (i) above, these converge in the Polish topology on $\operatorname{Aut}(X, \mu)$ and hence we have a transformation $\psi_{\gamma}: X \rightarrow X$ such that for all $A \subset X$ measurable,

$$
\mu\left(\gamma^{a_{n}} \cdot A \triangle \psi_{\gamma}(A)\right) \rightarrow 0 \quad \text { as } n \rightarrow \infty .
$$


Thus we obtain a fresh action $b \in A(\Gamma, X, \mu)$, with each $\gamma^{b}=\psi_{\gamma}$, such that for all $A \subset X$ measurable,

$$
\mu\left(\gamma^{a_{n}} \cdot A \triangle \gamma^{b} \cdot A\right) \rightarrow 0 \quad \text { as } n \rightarrow \infty .
$$

Note in particular that for all $A \in \mathcal{B}=\mathcal{B}_{0}$ and $\gamma \in F=F_{0}$ we have

$$
\mu\left(\gamma^{b} \cdot A \triangle \gamma^{a} \cdot A\right)<\varepsilon,
$$

and so it only remains to show the action is mixing.

Given any $n$ and $A, B \in \mathcal{B}_{n}$ and any $\delta>0$ we can find some $m>n$ with

$$
2^{-m-1} \varepsilon<\delta \text {. }
$$

Then for all $\gamma$ outside some finite set we have

$$
\left|\mu\left(A \triangle \gamma^{a_{m+2}} \cdot B\right)-\mu(A) \mu(B)\right|<\delta / 2
$$

by (iii). Then by (ii) we have, for all $k \geq m+2$,

$$
\left|\mu\left(A \triangle \gamma^{a_{k}} \cdot B\right)-\mu(A) \mu(B)\right|<\delta / 2,
$$

and hence

$$
\left|\mu\left(A \triangle \gamma^{b} \cdot B\right)-\mu(A) \mu(B)\right| \leq \delta / 2<\delta .
$$

Since $\bigcup_{n \in \mathbb{N}} \mathcal{B}_{n}$ is dense in $M(X, \mu)$, this suffices to show the action is mixing.

Acknowledgements. I am very grateful to Alexander Kechris for writing the monumentally informative [7], asking the question which resulted in this paper, and reading through an earlier draft and making a number of helpful suggestions.

I gratefully acknowledge support from the Australian Research Council in the form of an Australian Professorial Fellowship.

\section{References}

[1] V. Bergelson and J. Rosenblatt, Mixing actions of groups, Illinois J. Math. 32 (1988), 65-80.

[2] M. Burger, Kazhdan constants for SL(3,Z), J. Reine Angew. Math. 413 (1991), $36-67$.

[3] A. Connes and B. Weiss, Property T and asymptotically invariant sequences, Israel J. Math. 37 (1980), 209-210.

[4] E. Glasner and B. Weiss, Kazhdan's property $T$ and the geometry of the collection of invariant measures, Geom. Funct. Anal. 7 (1997), 917-935.

[5] P. de la Harpe et A. Valette, La propriété (T) de Kazhdan pour les groupes localement compacts, Astérisque 175 (1989).

[6] G. Hjorth and A. S. Kechris, Rigidity theorems for actions of product groups and countable Borel equivalence relations, Mem. Amer. Math. Soc. 177 (2005), no. 833.

[7] A. S. Kechris, Global aspects of ergodic group actions and equivalence relations, unpublished, http://www.math.caltech.edu/people/kechris.html. 
[8] D. Kerr and M. Pichot, Asymptotic Abelianness, weak mixing and property T, preprint.

[9] D. Ornstein and B. Weiss, Ergodic theory of amenable group actions. I. The Rohlin lemma, Bull. Amer. Math. Soc. (N.S.) 2 (1980), 161-164.

[10] A. L. T. Paterson, Amenability, Math. Surveys Monogr. 29, Amer. Math. Soc., Providence, RI, 1988.

[11] K. Schmidt, Amenability, Kazhdan's property T, strong ergodicity and invariant means for ergodic group-actions, Ergodic Theory Dynam. Systems 1 (1981), 223236.

Department of Mathematics and Statistics

University of Melbourne

Parkville, 3010

Melbourne, Victoria, Australia

E-mail: greg.hjorth@gmail.com

Received 31 May 2008;

in revised form 1 December 2008 